\title{
Preventing Parastomal Hernia with a Prosthetic Mesh: A 5-Year Follow-Up of a Randomized Study: Reply to Letter
}

\author{
Arthur Jänes · Leif Israelsson · Yucel Cengiz
}

Published online: 19 April 2009

(c) Société Internationale de Chirurgie 2009

Thank you for your comments [1] that illustrate several problems within the field of parastomal hernia [2]. Interpreting the calculations in your comment demands that the true rate of parastomal hernia in clinical practice is known, but unfortunately it is not. The rate of parastomal hernia is reported within the range of 5-65\% [3]. This probably reflects different definitions of parastomal hernia used at follow up and makes it very problematic to compare hernia rates between different reports. Actually, only a very few reports have presented the definition used. The proportion requiring repair is presumably also related to the definition of parastomal hernia used. The hernia rate seems to increase with time and the time to follow up in different reports then becomes crucial.

Surgeons may tend to recognize parastomal hernia as a problem mainly when a repair is demanded but this may not reflect the entire problem. We know that the definition used in our trial has clinical relevance and may reflect the reality faced by patients and stoma therapists [4]. We chose this definition as we also regarded it to be simple and reproducible.

The conclusion made by Dr Schafer [5] is founded on the fact that in the present trial the rate of parastomal hernia, with the definition used and presented, could be diminished with a prosthetic mesh without undue complications. The high rate of herniation in the non-mesh group in our trial cannot be an argument against the use of a

\footnotetext{
A. Jänes $(\bowtie) \cdot$ Y. Cengiz

Department of General Surgery, Kirurgkliniken,

Sundsvalls sjukhus, 85186 Sundsvall, Sweden

e-mail: arthur.janes@lvn.se

L. Israelsson

Department of Surgery and Perioperative Science,

Umeå University, Umea, Sweden
}

prophylactic mesh. The hernia rate in our trial simply cannot be compared with other reports if we do not know how parastomal hernia was then defined. Within a randomized trial the relation between hernia rates in patients receiving or not receiving a mesh is reliable with a constant definition of herniation used.

We believe that the low complication rate in our trial is related to the choice of prosthetic mesh and not to our department. Whether that is so and if results are reproducible is currently studied in a randomized multi-centre trial in Sweden. Further aspects on the definition of parastomal hernia at clinical and radiologic examination are currently studied at our department.

\section{References}

1. Evans MD, Williams GL, Stephenson BM (2009) Preventing parastomal herniation: Is prophylactic prosthetic mesh absolutely necessary? (Letter). World J Surg, doi:10.1007/s00268-0099972-y

2. Jänes A, Cengiz Y, Israelsson LA (2009) Preventing parastomal hernia with a prosthetic mesh: a 5-year follow-up of a randomized study. World J Surg 33:118-121

3. Israelsson LA (2008) Parastomal hernia. Surg Clin North Am 88(1):113-122

4. Kald A, Juul KN, Hjortsvang H, Sjödahl RI (2008) Quality of life is impaired in patients with peristomal bulging of a sigmoid colostomy. Scand J Gastroenterol 43(5):627-633

5. Schafer M (2009) Preventing parastomal hernia with a prosthetic mesh: a five year follow up of a randomized study (Invited Commentary). World J Surg 33:122-123 